\title{
Non-Surgical Rhinoplasty with Polydioxanone Threads and Hyaluronic Acid Filler to Treat a Widened Nose after Repeated Injections of Hyaluronic Acid Filler
}

\author{
Wook Oh, MD \\ Won Lee, $\mathrm{PhD}^{2}$ \\ ${ }^{1}$ Samsung Feel Clinic, Seoul, Korea \\ ${ }^{2}$ Yonsei E1 Plastic Surgery Clinic, Anyang, Korea
}

Received March 20, 2020

Revised April 9, 2020

Accepted April 13, 2020

\section{Correspondence}

Wook Oh

Samsung Feel Clinic, Seoul 06972, Korea

E-mail: feelclinic@naver.com

https://orcid.org/0000-0001-8319-2981

(C) Korean Society for Laser, Dermatology and Trichology

(c) This is an open access article distributed under the terms of the Creative Commons Attribution NonCommercial License (http://creativecommons.org/ licenses/by-nc/4.0) which permits unrestricted noncommercial use, distribution, and reproduction in any medium, provided the original work is properly cited.

\section{Background and Objectives}

Rhinoplasty with cosmetic filler is becoming an exceedingly popular, nonsurgical, cosmetic procedure, especially among Asians. However, the slim and pointed appearance achieved initially after treatment with filler injection tends to diminish over time, provoking complaints of a blunt shape and an overall enlarged nose due to flattening of the injected filler. This report describes the use of polydioxanone (PDO) threads and hyaluronic acid filler to correct a flattened, widen nose in two Korean women.

\section{Materials and Methods}

Two patients who had previously undergone treatment with filler injections presented with widened noses. Therein, we injected $150 \mathrm{IU}$ of hyaluronidase to dissolve remnant hyaluronic acid filler, and after 3 weeks, we inserted PDO threads and hyaluronic acid filler via non-surgical rhinoplasty.

Results

In both patients, hyaluronidase dissolved previous filler that had begun to disintegrate and lead to a widened nose. Subsequent treatment with PDO threads and hyaluronic acid filler restored the desired appearance of a slim and pointed nose. No adverse side-effects from either treatment were reported.

\section{Conclusion}

The use of hyaluronidase and non-surgical rhinoplasty with PDO threads and hyaluronic acid filler are useful in the correction of a widened nose created by repeated injections of hyaluronic acid filler.

Key words

Rhinoplasty, PDO threads, Hyaluronic acid filler 


\section{INTRODUCTION}

Rhinoplasty with cosmetic filler is an exceedingly popular, non-surgical, cosmetic procedure, especially among Asians. The cosmetics effects achieved with fillers, however, persist for only a limited duration, although many manufacturers of cosmetic fillers ensure that their products provide long lasting effects of up to 2 to 3 years. With the short maintenance period and a need for frequent re-treatment to maintain the desired shape, rhinoplasty with cosmetic filler garners relatively low satisfaction among patients and physicians. Interestingly, one study of hyaluronic acid filler reported long-term maintenance of the filler up to 64 weeks in rats [1]. Unfortunately, this study merely confirmed the presence of the filler at injection sites, and the maintenance assessment did not consider cosmetic effects in actual patients: the maintenance duration likely would not be as long if the results reflected visual symmetry and patient satisfaction with their appearance. Meanwhile, another report indicated that hyaluronic acid filler injected into the nasolabial fold provided clinically satisfactory results for up to 52 weeks [2]. While these results are promising, rhinoplasty with dermal filler is markedly different from filler procedures in other areas, such as the forehead, cheeks, temples, and nasolabial folds, as the filler must be placed precisely in a narrow area to achieve a high, pointed nose, rather than being used to augment a relatively wide area.

Despite the somewhat short maintenance period, injected filler generally does not completely disappear between treatments. Research has demonstrated that some of the injected filler is replaced with autologous tissue via fibroblast proliferation, collagen induction, and angiogenesis, followed by the proliferation of adipocytes, providing maintenance of the initially injected volume [1]. These results would be welcomed for filler treatments on the forehead, cheeks, and temples, but not on the nose, where the maintenance of both volume and shape are crucial. Moreover, having lost the slim and pointed appearance achieved initially after treatment, patients who undergo repeated rhinoplasties with filler injection often present with complaints of a blunt shape and an overall enlarged nose due to flattening of the injected filler.

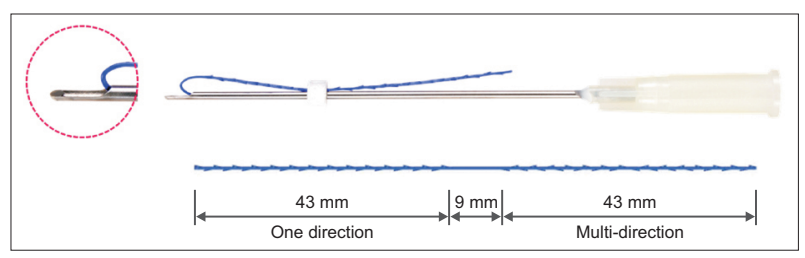

Fig. 1. Schematic diagram of MINT Lift ${ }^{\circledR}$ UP.
This report describes the use of hyaluronidase to dissolve remnant filler injected in the nose and a re-treatment procedure using polydioxanone (PDO) threads and hyaluronic acid filler to achieve satisfactory treatment results in two Korean women.

\section{MATERIALS AND METHODS}

\section{Polydioxanone (PDO) threads}

MINT Lift ${ }^{\circledR}$ UP (Fig. 1) and MINT Lift ${ }^{\circledR}$ BIJOU PDO threads (Fig. 2) (HansBioMed, Daejeon, Republic of Korea) were used in this study. The threads are composed of PDO at a USP size of 2-0, an average diameter of 0.300-0.339 mm, and lengths of $95 \mathrm{~mm}$ for MINT Lift ${ }^{\circledR}$ UP threads and $40 \mathrm{~mm}$ for MINT Lift ${ }^{\circledR}$ BIJOU threads. As shown in Fig. 1 and Fig. 2, the threads are designed to be inserted to half the length of the thread via a 19G L-type cannula with a diameter of $1.2 \mathrm{~mm}$. The half of the thread placed inside the cannula consists of multiple bi-directional barbs; the other half comprises one-directional barbs.

\section{Dermal filler and hyaluronidase}

For use as dermal filler in this study, Amalian SF $24{ }^{\circledR} \mathrm{Ad}$ vanced filler (S\&V Technologies, Hennigsdorf, Germany) was obtained from Daehwa Pharmaceutical (Seoul, Republic of Korea). The hyaluronic acid filler comprises a monophasic filler containing $24 \mathrm{~g} / \mathrm{ml}$ of hyaluronic acid. Liporase ${ }^{\circledR}$ hyaluronidase (1,500 IU; Daehan New Pharm Co., Hwaseong, Republic of Korea) was obtained for use in dissolving remnant hyaluronic acid dermal filler in the target area.

\section{Patients}

Case 1 involved a 27-year-old female patient who had undergone four hyaluronic acid filler injections on the nose over the previous 5 years prior to the visiting our clinic (Fig. 3). Case 2 comprised a 24-year-old female patient who had undergone two prior injections of hyaluronic acid filler on the nose over the previous 2 years (Fig. 4). In both patients, the nose appeared blunted due to a widened and thick nasal dorsum stemming from several prior filler injections. Both patients underwent treatment with hyaluronidase in-

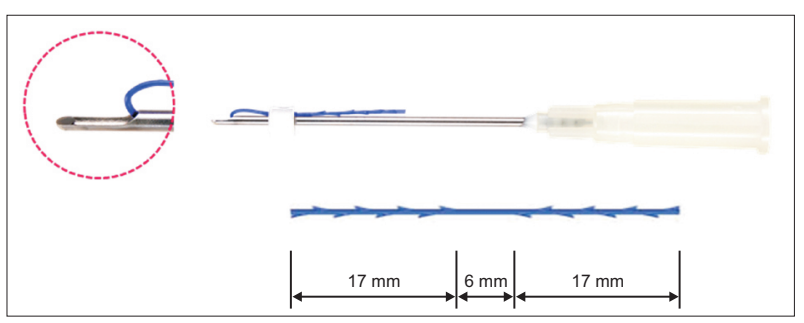

Fig. 2. Schematic diagram of MINT Lift ${ }^{\circledR}$ BIJOU. 
 \\ AESTHETICS}
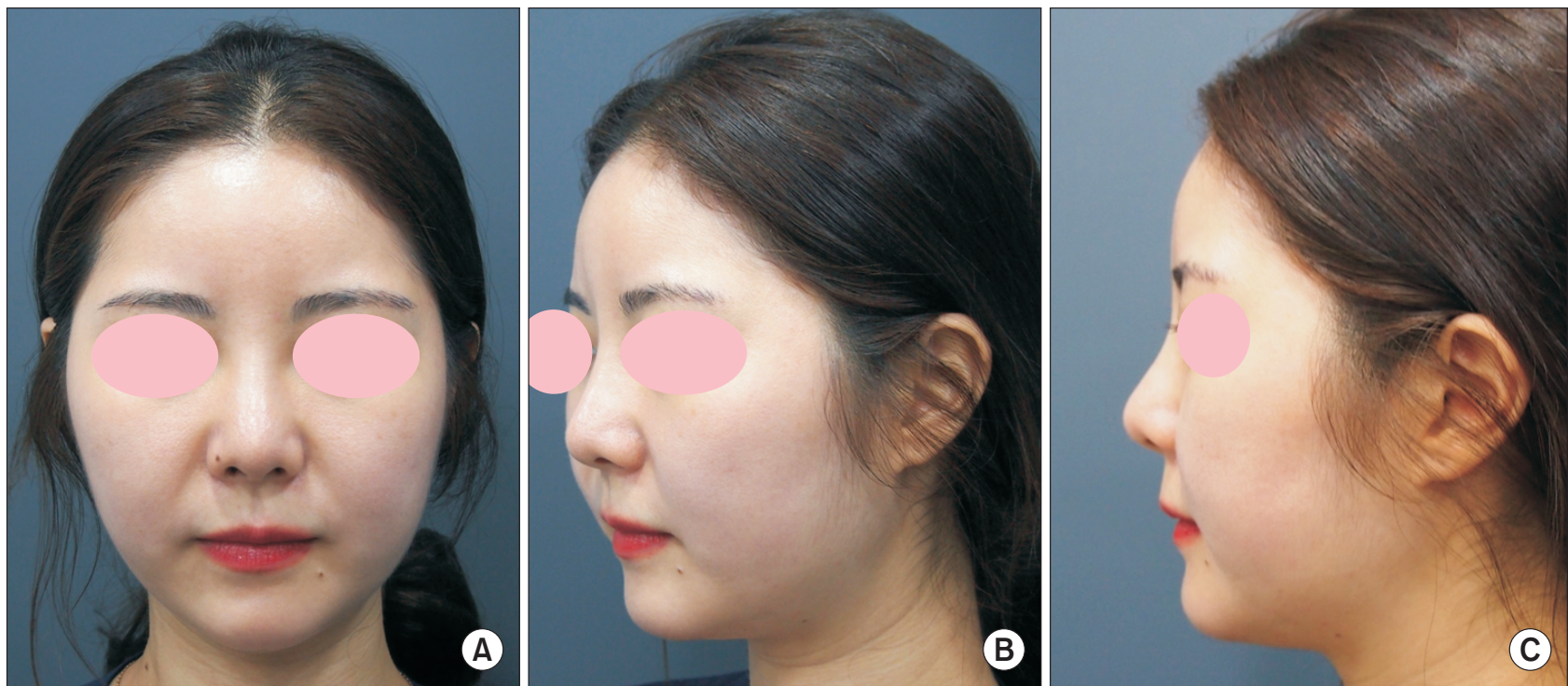

Fig. 3. Case 1. A 27-year-old female patient who had undergone four hyaluronic acid filler injections on the nose over 5 years. (A) Front view before treatment. (B) Three-quarter view before treatment. (C) Lateral view before treatment.
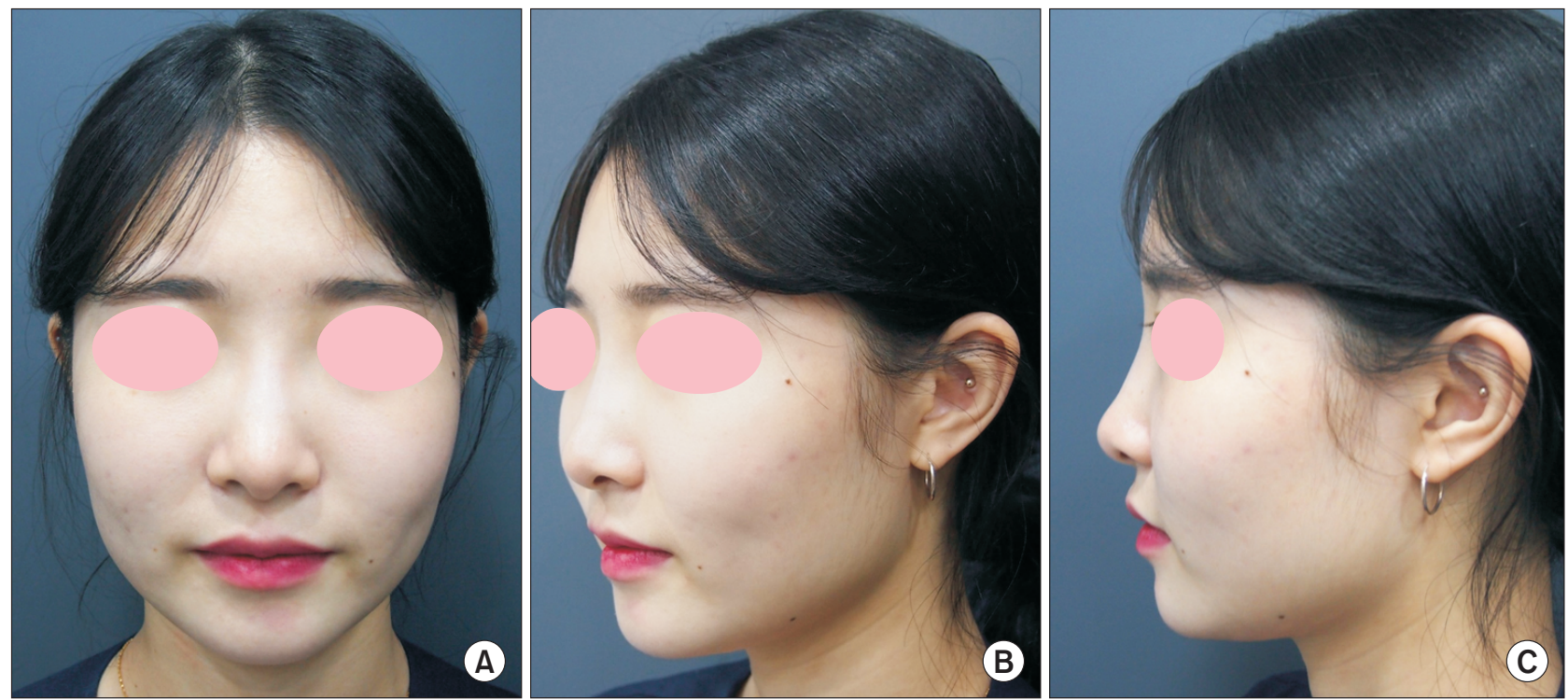

Fig. 4. Case 2. A 24-year-old female patient who had undergone two prior injections of hyaluronic acid filler on the nose over the previous 2 years. (A) Front view before treatment. (B) Three-quarter view before treatment. (C) Lateral view before treatment.

jections to dissolve any hyaluronic acid dermal filler present and rhinoplasty with PDO threads and hyaluronic acid filler, as described in the following sections.

\section{Surgical procedures}

\section{1) Hyaluronidase injection}

Prior to the rhinoplasty, $150 \mathrm{IU}$ of hyaluronidase (Liporase $^{\circledR}$; Daehan New Pharm Co., Hwaseong, Republic of Korea) was prepared and injected at three points along the nasal dorsum and glabella. Following the injections, the area was gently massaged to help promote local diffusion. No hyaluronidase was injected in the nasal tip or columella, as there was no palpable filler present. The hyaluronidase injection site was then allowed to recover for 1 week, after which no filler component along the target injection site was palpable, and no further injections of hyaluronidase were required. Neither patient showed any side effects related with the hyaluronidase injections. After an additional 2 weeks, we confirmed that the results were no different to 

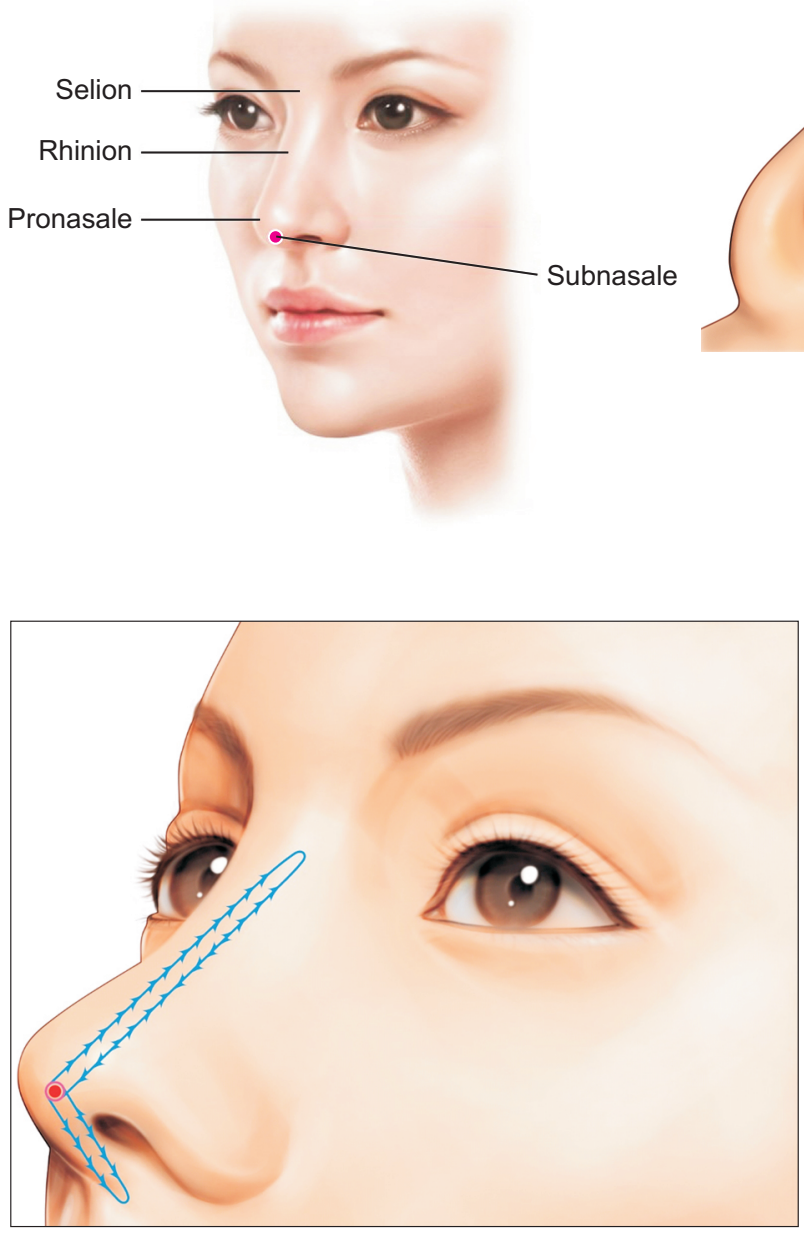

Fig. 6. MINT Lift ${ }^{\circledR}$ UP and MINT Lift ${ }^{\circledR}$ BIJOU threads inserted along the nasal dorsum and the columella.

those observed after week 1 and continued with the scheduled rhinoplasty.

\section{PDO thread insertion and dermal filler injection}

Prior to the rhinoplasty procedure, lidocaine $2 \%$ was utilized to achieve an infraorbital nerve block on both sides of the face and local anesthesia of the glabella and nasal tip. A site along the subnasale area was then punctured with a $18 \mathrm{G}$ needle for introduction of a cannula (Fig. 5). The puncture site was made at a site that did not affect the pronasale area, as traces of the puncture site can be seen for more than a month after the procedure and as inflammation can cause pigmentation that does not readily disappear.

After creating the puncture site, MINT Lift ${ }^{\circledR}$ UP threads were gently placed via a cannula inserted through the puncture site deep into the glabella, reaching the selion area. The cannula was then removed while slightly pressing on the end thereof to prevent the PDO thread from being pulled out with the cannula (Fig. 6). A total of four threads
Fig. 5. Anthropologic landmarks on the nose and recommend puncture site (red dot).

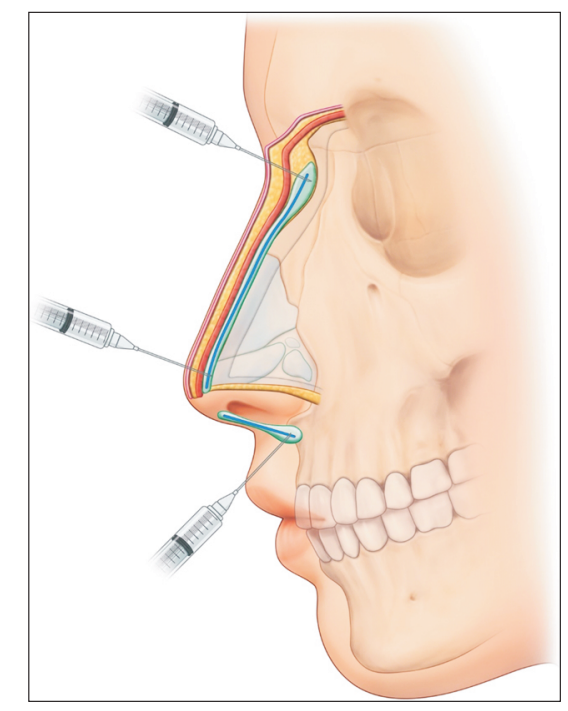

Fig. 7. Schematic diagram of hyaluronic acid filler injection.

were inserted. Next, additional MINT Lift ${ }^{\circledR}$ BIJOU threads were inserted along the columella via a cannula positioned deep enough to touch the anterior spine of maxillary bone. After inserting four threads, we confirmed that none pressed against the gums or had breached the upper gum area into the patient's mouth.

Upon completing the insertion of the PDO threads, hyaluronic acid filler was injected to create a slimmer nose and to achieve the desired augmentation (Fig. 7). The filler was injected directly into the target area by piercing the skin perpendicular to the threads, although it can also be injected via the same cannula and puncture site used to introduce the PDO threads. We ensured that the filler was injected into either the supraperiosteal layer or supraperichondrial layer, and we were careful not to inject a large amount of filler at the pronasale area, as this may cause leakage from the puncture site where the threads are inserted. At 2 weeks after the filler procedure, the shape of 
 \\ AESTHETICS}

the nose was evaluated, and if necessary, additional filler was injected to achieve the desired appearance.

\section{RESULTS}

Fig. 8 and Fig. 9 depict the appearances of the patients in Cases 1 and 2, respectively, before the procedure, at 1 week after the hyaluronidase injection, and after insertion of the PDO threads and hyaluronic acid filler. In the side profile pictures taken prior to the hyaluronidase injections, the glabella area is raised; however, from the front, the area lacks sharpness and appears unattractive and blunt due to several prior filler injections that have widened and thickened the nasal dorsum. In the pictures taken after the hyal- uronidase injections, which dissolved the filler between the glabella, the nasal dorsum appears much lower. Additionally, although the side profile pictures taken immediately after the rhinoplasty (Fig. 8C3, 9C3) show no marked difference in the height of the nasal dorsum, compared to those before the procedure (Fig. 8A3, 9A3), the width of the nasal dorsum after the procedure (Fig. $8 \mathrm{Cl}, 9 \mathrm{C} 1$ ) is much narrower than that prior to the procedure (Fig. 8A1, 9A1) on the frontal view. This provided a much sharper appearance with which both patients were highly satisfied. Also, no side effects or problems with the hyaluronidase injections, thread insertion, or filler injections were recorded.

Fig. 10 and Fig. 11 comprise pictures of the same patients before and 6 months after the rhinoplasty. The shape
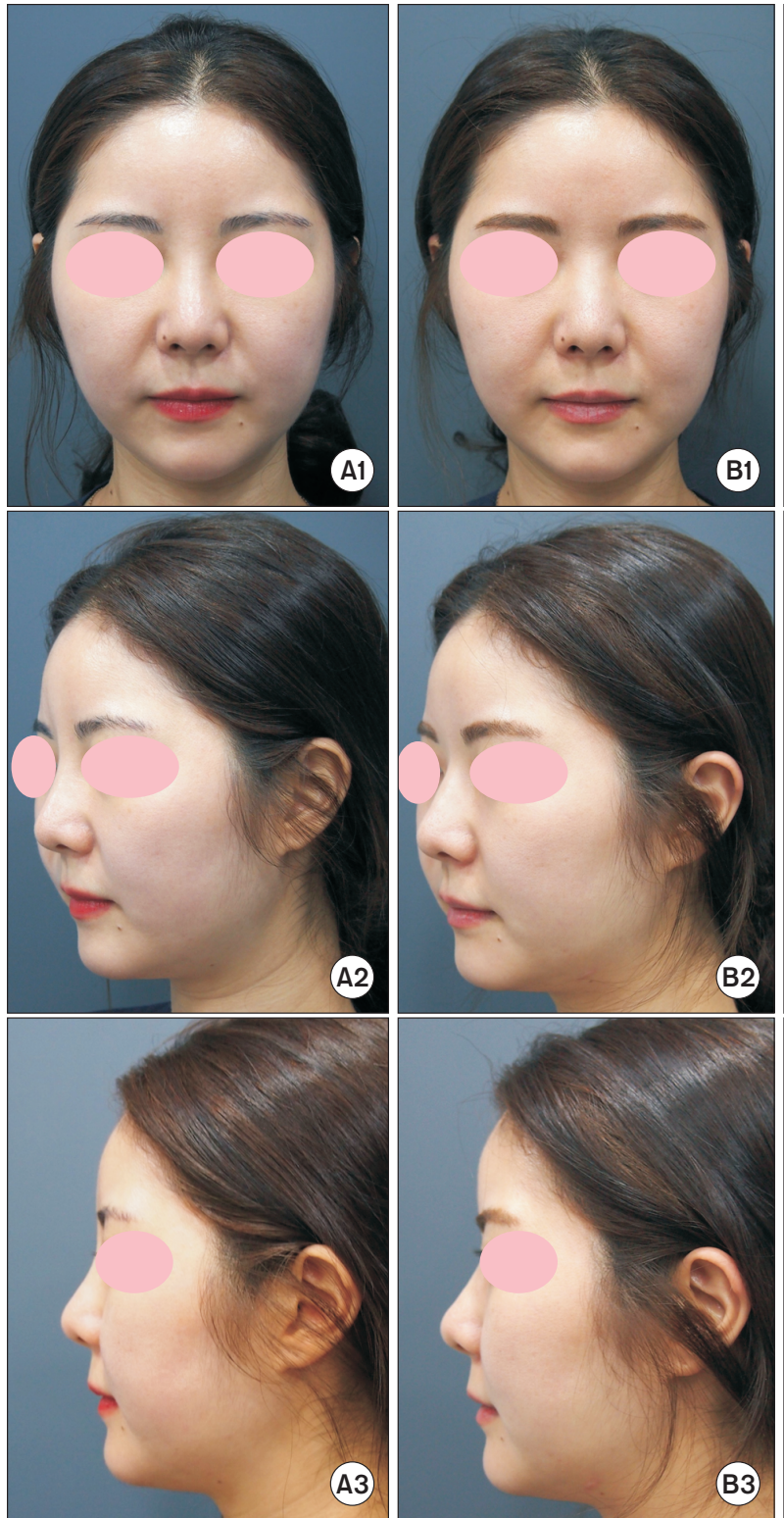

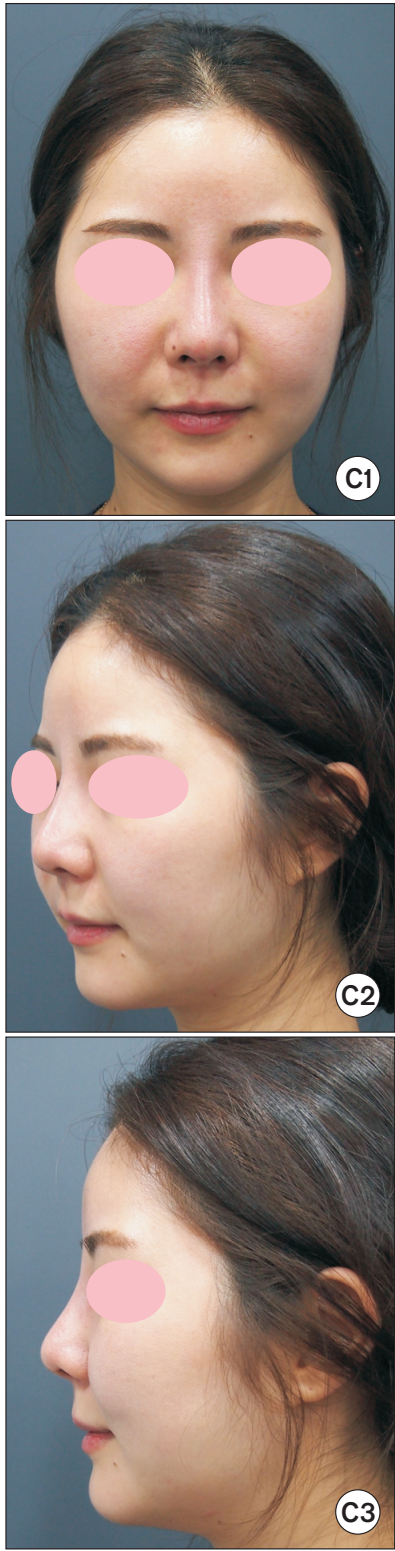

Fig. 8. Photographs of the patient in Case 1 before (A1-3), 1 week after hyaluronidase injection (B1-3), and immediately after the injection of PDO threads and hyaluronic acid filler (C1-3). 

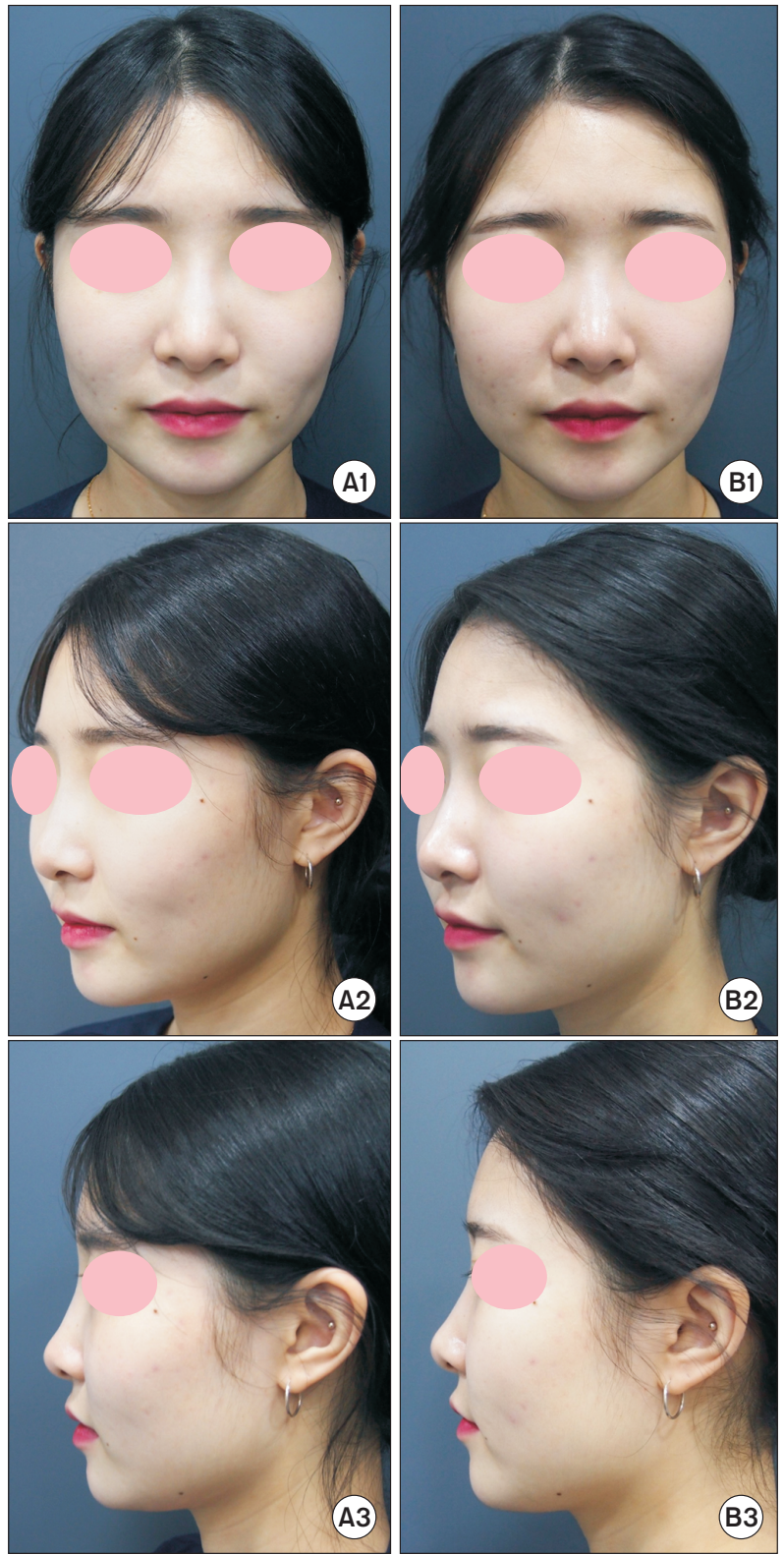

B3.
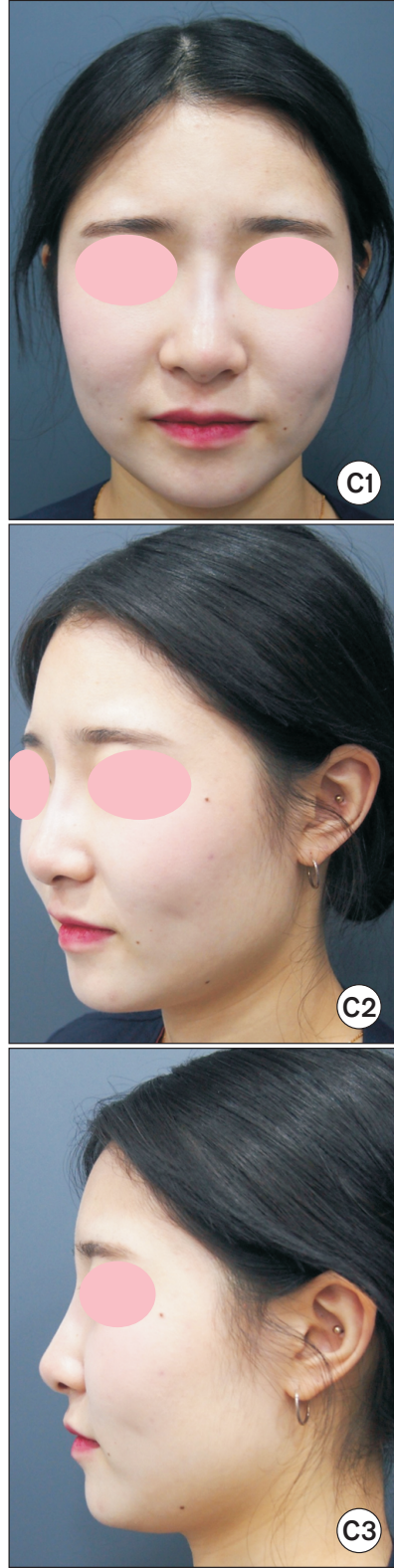

Fig. 9. Photographs of the patient in Case 2 before (A1-3), 1 week after hyaluronidase injection (B1-3), and immediately after the injection of PDO threads and hyaluronic acid filler (C1-3). of the nose at 6 months after the procedure remained narrow, straight, and attractive, and exhibited very little change from the shape achieved immediately after the procedure. Both patients reported much greater satisfaction with their appearance at 6 months after treatment with hyaluronidase and rhinoplasty with PDO threads and filler than with that after multiple filler procedures over the previous years.

\section{DISCUSSION}

Filler treatments are attractive procedures that many patients choose to undergo despite their short maintenance period, and patients who have once been treated with a filler procedure often undergo two to three additional follow-up treatments, seeking a more marked change in their appearance. While studies have not yet surveyed patient expectations for re- or multiple treatments, most patients generally expect to see additional benefits and maintenance of previous results with additional treatments. Meanwhile, a decrease in volume can lead some to overestimate the actual amount of filler lost, and in result, both the patient and clinician may seek to increase the volume of filler beyond what was lost in re-treatments, leading to an undesired appearance.

Although conducted with rats, the study by Mochizuki et al. [1] demonstrated that the total increase in volume achieved with an injection of hyaluronic acid filler remained after 64 weeks, despite a slight flattening of the bolus injec- 


\section{AESTHETICS}

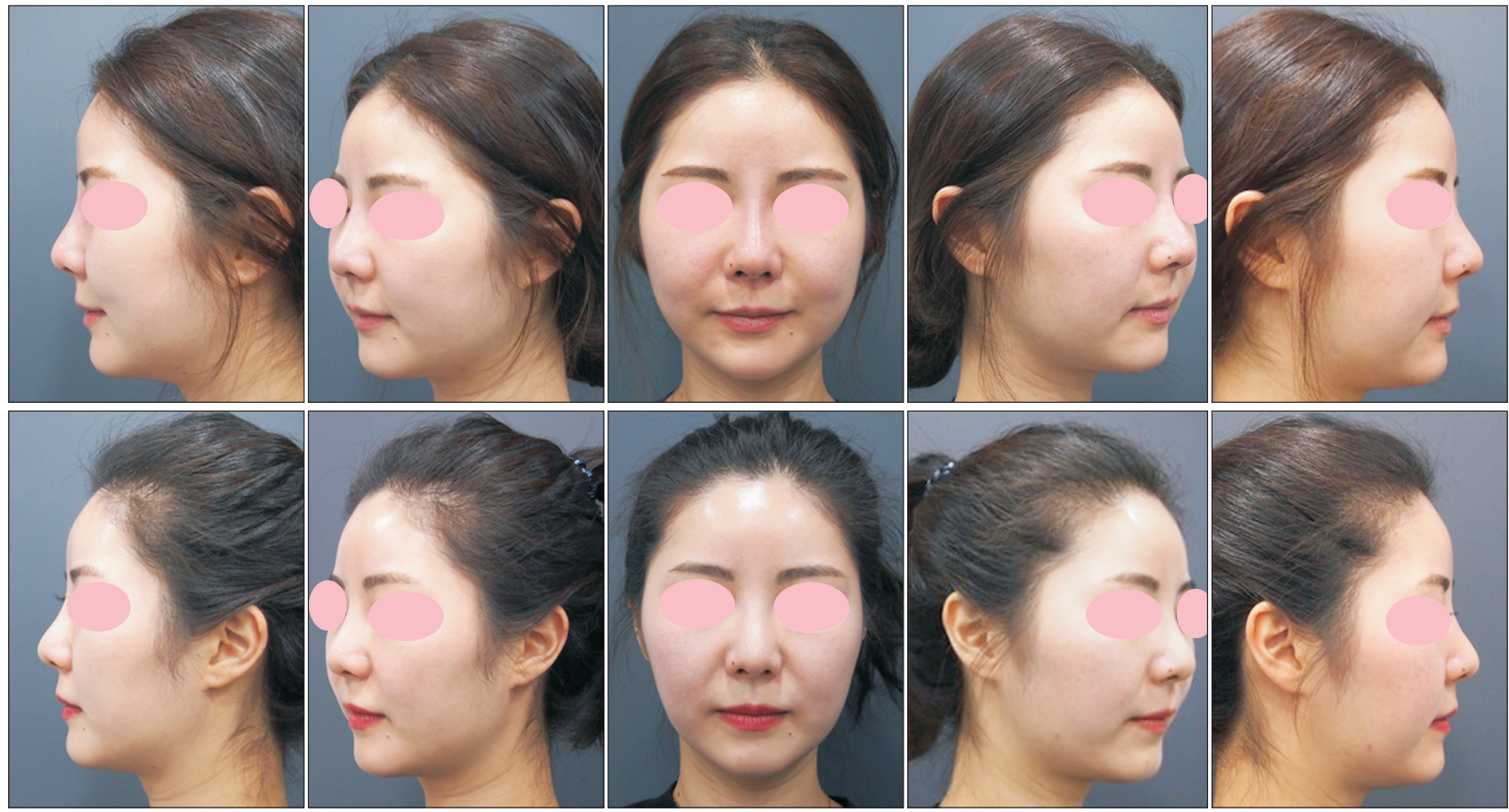

Fig. 10. Photographs of the patient in Case 1 immediately after the injection of PDO threads and hyaluronic acid filler (above) and 6 months after the procedure (below).
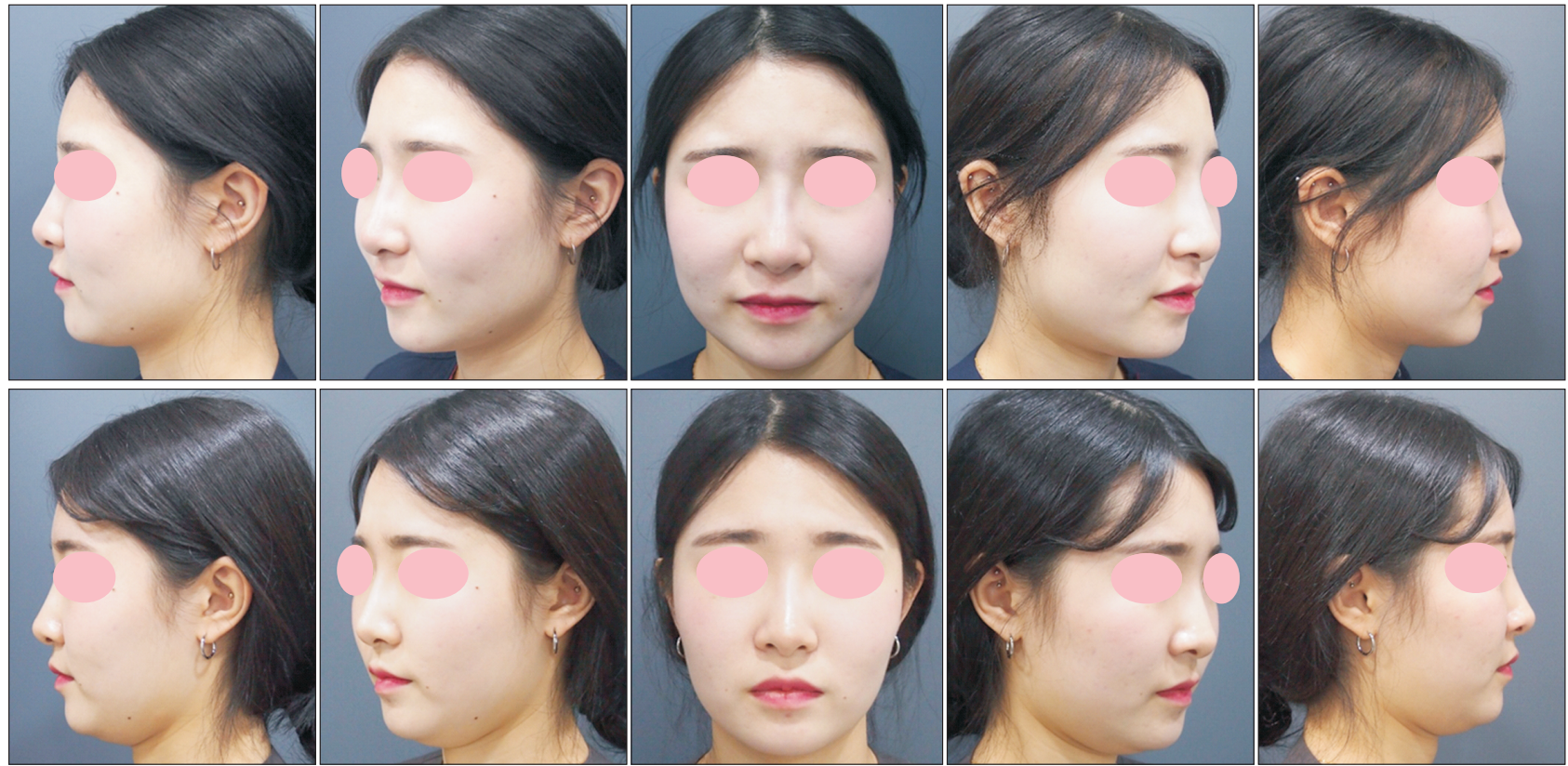

Fig. 11. Photographs of the patient in Case 2 immediately after the injection of PDO threads and hyaluronic acid filler injection (above) and 6 months after the procedure (below).

tion. The study indicated that some of the filler component acted as a scaffold for autologous tissue replacement, thereby providing longer maintenance of the initial injected volume for up to 64 weeks. While these results are encouraging, further consideration must be given in regards to the characteristics of the target site and the expected clinical outcome. For example, filler treatments along the forehead or temples do not necessarily require the filler to remain in its first injected position, so long as the volume is maintained. However, along the nose, any flattening or 
slight migration of injected filler would be unwanted and necessitate more frequent re-treatment. As such, clinicians often inject additional filler when previous filler has not yet disappeared, leading to an excessive increase in volume and a blunt or enlarged nose.

In filler rhinoplasty, a large amount of filler is, in addition to the nasal dorsum, also injected in the intercanthal area, especially when treating Asian patients. In the study by Kim and Ahn [3], the authors recommended augmentation of the nasal dorsum during filler rhinoplasty until the new radix level reached the supratarsal crease. Jasin [4], on the other hand, was concerned with and opposed raising the new radix level this high. The authors insisted that the level of the ciliary margin of the upper lid or slightly below was more appropriate, with which we agree. Nonetheless, such claims are made because the large amount of filler injected in the nasal dorsum area can lead to problems in the long run: flattening of the filler over time necessitates additional filler procedures to achieve a heightened appearance on the side profile that otherwise seems blunt and unattractive from the front, as encountered in the two cases presented in this report.

The objective of filler rhinoplasty is to make the nose appear narrow. To do so, Jasin [4] argued that it is important to inject filler into the midline and to avoid injections on either side thereof, as they would widen the appearance of the nose. However, as observed in the two cases in this report, additional filler injections along the midline of an already widened nose due to previous filler procedures can worsen the appearance over time. To treat such cases, we found that dissolving any previously injected filler with hyaluronidase and then replacing it with new filler provided satisfactory outcomes. However, the same problem with a widening nose would likely occur in the future without additional effort to address it appropriately.

When dissolving previous filler with hyaluronidase in order to inject fillers or PDO threads, the timing of the procedures requires attention, as the injection of hyaluronic acid fillers can be affected by the injection of hyaluronidase prior thereto. Kim et al. [5] demonstrated that the effects of hyaluronidase dissipate within 3-6 hours post injection and that reinjection with hyaluronic acid fillers can be undertaken after 6 hours of using hyaluronidase. However, it has been reported that hyaluronidase can degrade hyaluronic acid filler for up to 30 days or more. Therefore, further study is needed to determine a safe point at which hyaluronic acid filler can be injected after treatment with hyaluronidase to ensure that the treated area and resultant appearance is not affected. While, understandably, patients would hope to be treated as soon as possible after the hyaluronidase injection, clinicians must be sure that hyaluronidase will not later affect the injected threads and filler. After much deliberation, we chose to apply a waiting period of 3 weeks, although more discussion and research thereof is needed.

Several studies have documented the use of threads in rhinoplasty. Lee and Yang [6] demonstrated that the use of threads in rhinoplasty achieved excellent results in lifting of the nasal tip. Kang et al. [7] also reported that PDO threads were especially useful for the lifting of the tip of the nose and straightening of the columella-labial angle for a more satisfying and balanced appearance. Meanwhile, Jung [8] noted that when injecting both PDO threads and hyaluronic acid fillers, the threads acted as a scaffold for the filler and provided longer retention of the filler, compared to procedures with fillers alone. Moreover, the filler inhibited chemical reactions between PDO threads and water, thereby extending the life of the threads. For this reason, we injected filler around the PDO threads in the patients described in this report.

The potential side effects of filler procedures vary greatly in number and severity, from skin necrosis to blindness. However, there were no cases of fatal side effects caused by PDO threads in previous reports. As such, adding threads to a filler procedure, which can minimize the amount of filler required to achieve the desired treatment endpoint, may help to reduce the likelihood of adverse side effects occurring. Despite these positive results, many plastic surgeons have concerns about whether the injection of threads and fillers together will have a negative impact on the treated area should the patient might require open surgery in the future. Recently, there was a report of infection by PDO threads [9]. Unlike this report case, our study is performed relatively small amount of threads and performed absolute aseptic conditions. So doctors always should consider possible infections and should perform absolute aseptic conditions. Accordingly, long-term follow-up studies of the procedure is needed.

\section{CONCLUSION}

Rhinoplasty with PDO threads and hyaluronic acid filler has many advantages, including a longer maintenance period and less migration or flattening of the filler. Also, the use of threads facilitates lifting of the nasal tip and columella. In this report, we found the use of hyaluronidase followed by rhinoplasty with PDO threads and hyaluronic acid filler to be effective in alleviating the appearance of a widened, blunt nose caused by repeated rhinoplasties with filler in two Korean women. 


\section{The \\ AESTHETICS}

\section{CONFLICT OF INTEREST}

Wook Oh has been an investigator, speaker, and consultant for Daehwa Pharmaceutical and HansBioMed Co., LTD. Amalian SF $24^{\circledR}$ Advanced hyaluronic acid filler was provided by Daehwa Pharmaceutical, Republic of Korea. MINT Lift $^{\circledR}$ UP and MINT Lift ${ }^{\circledR}$ BIJOU PDO threads was provided by HansBioMed Co., LTD., Republic of Korea.

\section{REFERENCES}

1. Mochizuki M, Aoi N, Gonda K, Hirabayashi S, Komuro Y. Evaluation of the in vivo kinetics and biostimulatory effects of subcutaneously injected hyaluronic acid filler. Plast Reconstr Surg 2018;142:112-21.

2. Kwon HJ, Ko EJ, Choi SY, Choi EJ, Jang YJ, Kim BJ, et al. The efficacy and safety of a monophasic hyaluronic acid filler in the correction of nasolabial folds: a randomized, multicenter, single blinded, split-face study. J Cosmet Dermatol 2018;17:584-9.
3. Kim P, Ahn JT. Structured nonsurgical Asian rhinoplasty. Aesthetic Plast Surg 2012;36:698-703.

4. Jasin ME. Nonsurgical rhinoplasty using dermal fillers. Facial Plast Surg Clin North Am 2013;21:241-52.

5. Kim HJ, Kwon SB, Whang KU, Lee JS, Park YL, Lee SY. The duration of hyaluronidase and optimal timing of hyaluronic acid (HA) filler reinjection after hyaluronidase injection. J Cosmet Laser Ther 2018;20:52-7.

6. Lee HY, Yang HJ. Rhinoplasty with barbed threads. Plast Reconstr Surg Glob Open 2018;6:e1967.

7. Kang SH, Moon SH, Kim HS. Nonsurgical rhinoplasty with polydioxanone threads and fillers. Dermatol Surg. Forthcoming 2019. https://doi.org/10.1097/DSS.0000000000002146

8. Jung GS. Minimally invasive rhinoplasty technique using a hyaluronic acid filler and polydioxanone threads: an effective combination. Facial Plast Surg 2019;35:109-10.

9. Kim HJ, Lee SJ, Lee JH, Kim SH, Suh IS, Jeong HS. Clinical features of skin infection after rhinoplasty with only absorbable thread (polydioxanone) in oriental traditional medicine: a case series study. Aesthetic Plast Surg 2020;44:139-47. 